\title{
24. COCCOLITH ZONATION OF CORES FROM THE WESTERN INDIAN OCEAN AND THE GULF OF ADEN, DEEP SEA DRILLING PROJECT LEG $24^{1}$
}

\author{
David Bukry, United States Geological Survey, La Jolla, California
}

Leg 24 of the Deep Sea Drilling Project, May-June 1972, began at Djibouti, near the Gulf of Aden, and ended at Port Louis, east of Madagascar (Figure 1). This leg investigated

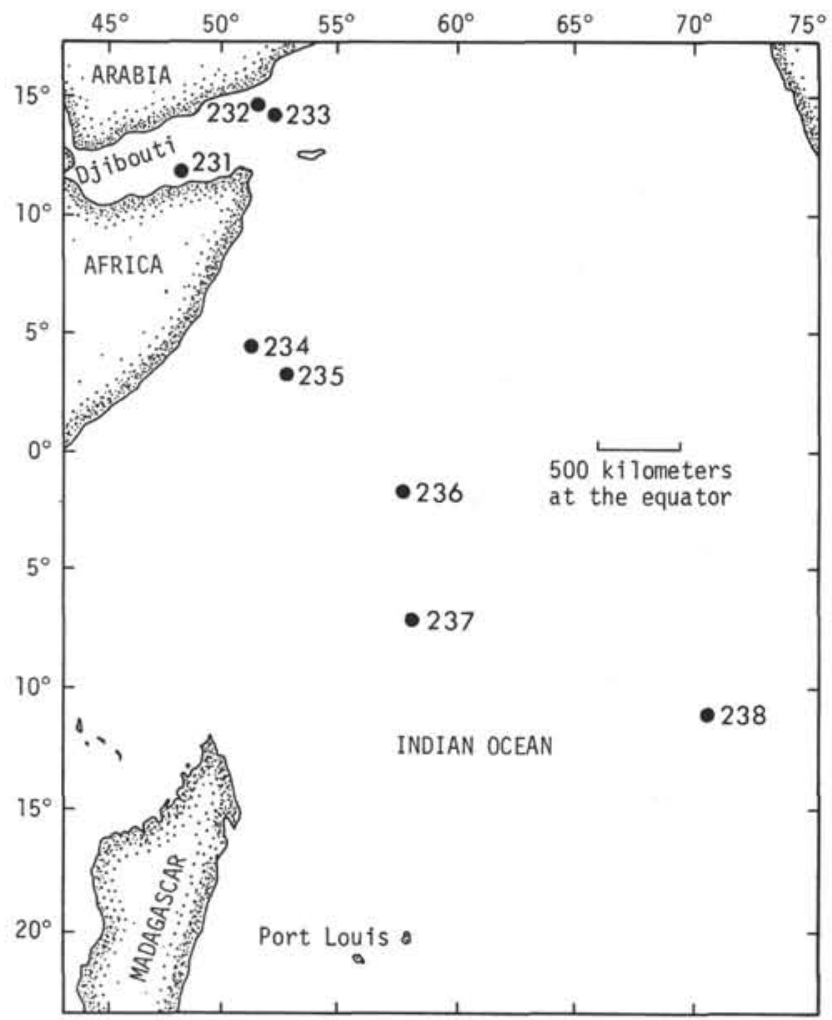

Figure 1. Sketch map showing Leg 24 sites in the western Indian Ocean and the Gulf of Aden.

\footnotetext{
${ }^{1}$ Publication authorized by the Director, U.S. Geological Survey.
}

the western Indian Ocean and recovered 337 cores at eight drilling sites (Table 1). Light-microscope techniques were used to study the coccoliths of 305 samples from these cores. The zonation employed in zonal assignments of core samples from Leg 24, summarized in Figure 2, is based on Bukry (1973). The sediment is primarily coccolithic chalk and marl (Fisher et al., 1972).

\section{REFERENCES}

Bukry, D., 1973. Low-latitude coccolith biostratigraphic zonation. In Edgar, N. T., Saunders, J. B., et al., Initial Reports of the Deep Sea Drilling Project, Volume 15: Washington, (U.S. Government Printing Office), p. 685-703.

Fisher, R. L., Bunce, E. T., Cernock, P. J., Clegg, D. C., Cronan, D. S., Damiani, V. V., Dmitriev, L. V., Kinsman, D. J. J., Roth, P. H., Thiede, J., and Vincent, E. S., 1972. Deep Sea Drilling Project in dodo land Leg 24: Geotimes, v. 17, p. 17-21.

TABLE 1

Location, Water Depth, Penetration, and Number of Cores Cut at Deep Sea Drilling Project Sites in the Gulf of Aden and Western Indian Ocean

\begin{tabular}{|c|c|c|c|c|c|}
\hline Site & Latitude & Longitude & $\begin{array}{l}\text { Water } \\
\text { Depth } \\
\text { (m) }\end{array}$ & $\begin{array}{l}\text { Penetration } \\
\text { (m) }\end{array}$ & Cores \\
\hline \multicolumn{6}{|c|}{ Gulf of Aden } \\
\hline 231 & $11^{\circ} 53.41^{\prime} \mathrm{N}$ & $48^{\circ} 14.71^{\prime} \mathrm{E}$ & 2161 & 584 & 64 \\
\hline 232 & $14^{\circ} 28.93^{\prime} \mathrm{N}$ & $51^{\circ} 54.87^{\prime} \mathrm{E}$ & 1758 & 434 & 49 \\
\hline 233 & $14^{\circ} 19.68^{\prime} \mathrm{N}$ & $52^{\circ} 08.11^{\prime} \mathrm{E}$ & 1860 & 271 & 32 \\
\hline \multicolumn{6}{|c|}{ Western Indian Ocean } \\
\hline 234 & $04^{\circ} 28.96^{\prime} \mathrm{N}$ & $51^{\circ} 13.48^{\prime} \mathrm{E}$ & 4738 & 277 & 16 \\
\hline 235 & $03^{\circ} 14.06^{\prime} \mathrm{N}$ & $52^{\circ} 41.64^{\prime} \mathrm{E}$ & 5146 & 684 & 20 \\
\hline 236 & $01^{\circ} 40.62^{\prime} \mathrm{S}$ & $57^{\circ} 38.85^{\prime} \mathrm{E}$ & 4504 & 328 & 37 \\
\hline 237 & $07^{\circ} 04.99^{\prime} \mathrm{S}$ & $58^{\circ} 07.48^{\prime} \mathrm{E}$ & 1640 & 694 & 67 \\
\hline 238 & $11^{\circ} 09.21^{\prime} \mathrm{S}$ & $70^{\circ} 31.56^{\prime} \mathrm{E}$ & 2844 & 587 & 64 \\
\hline
\end{tabular}




\begin{tabular}{|c|c|c|c|c|c|c|c|c|c|c|}
\hline $\begin{array}{l}\text { Series or } \\
\text { Subseries }\end{array}$ & Zone & Subzone & 231 & 232 & 233 & 234 & 235 & 236 & 237 & 238 \\
\hline Holocene & Emiliania huxleyi & & & & & & & $1-2$ & $1-1$ & \\
\hline \multirow{3}{*}{ Pleistocene } & Gephyrocapsa oceanica & & $1-1 / 9 \cdot 3$ & $2-4 / 5-4$ & 1.2 & & 1.2 & & $? 2-1$ & $1.1 / 1-4$ \\
\hline & Crenalithus doronicoides & Gephyrocapsa caribbeanica & $10-3$ & \multirow{2}{*}{$7-2 / 9-2$} & $3-2 / 5-4$ & & \multirow{2}{*}{$2 \cdot 2 / 4-3$} & & & 3.2 \\
\hline & Crenalithus doronicoides & Emitiania annula & $11-2 / 12-3$ & & $7 \cdot 5 / 9-2$ & & & $3-2$ & & $6-2$ \\
\hline \multirow{3}{*}{$\begin{array}{l}\text { Upper } \\
\text { Pliocene }\end{array}$} & \multirow{3}{*}{ Discoaster brouweri } & Cyclococcolithina macintyrei & $13-3 / 15-2$ & $10-2 / 11-3$ & $10-2 / 13-2$ & & $5-2$ & & & $7 \cdot 2$ \\
\hline & & Discoaster pentaradiatus & $17-3 / 19-3$ & $13-1 / 15-3$ & $15-2 / 5 \mathrm{~A}-2$ & $1-1$ & & & & $8-2$ \\
\hline & & Discoaster tamalis & & $16-3$ & & $i-3$ & $7-3$ & & $4-2 / 4-5$ & $10-5 ; 4-5$ \\
\hline \multirow{4}{*}{$\begin{array}{l}\text { Lower } \\
\text { Pliocene }\end{array}$} & \multirow{2}{*}{$\begin{array}{l}\text { Reticulofenestra } \\
\text { pseudoumbilica }\end{array}$} & Discoaster asymmetricus & \multirow{2}{*}{$21-3 / 27-3$} & \multirow{2}{*}{$17-3 / 7 \mathrm{~A}-2$} & & & & & $5-5$ & $4-2 / 5-2$ \\
\hline & & Sphenolithus neoabies & & & & & & & $6-5 / 7-5$ & $11-5 / 12-5$ \\
\hline & \multirow{3}{*}{ Ceratolithus tricorniculatus } & Ceratolithus rugosus & & & & & $9-2$ & $4-3$ & $8-5$ & $13-5$ \\
\hline & & Ceratolithus acutus & $28-3$ & $9 A-5$ & & & & & $9-2$ & $15-5$ \\
\hline \multirow{5}{*}{$\begin{array}{l}\text { Upper } \\
\text { Miocene }\end{array}$} & & Triquetrorhabdulus rugosus & $? 29-3$ & $? 10 \mathrm{~A}-4 / 19 \mathrm{~A}-1$ & & & & $? 5-4 / 6-2$ & $? 9-5$ & $16-2$ \\
\hline & \multirow{2}{*}{ Discoaster quinqueramus } & Ceratolithus primus & \multirow{2}{*}{$30-3 / 45-3$} & \multirow{2}{*}{$21 \mathrm{~A}-2 / 24 \mathrm{~A}-1$} & & & $10-2 / 11-2$ & $6-5 / 12-5$ & $10-2 / 10-5$ & $16-5 / 19 \cdot 5$ \\
\hline & & Discoaster berggrenit & & & & & & $13-5$ & $11-5 / 12-5$ & $20-5 / 23-5$ \\
\hline & Discogster neohamatus & Discoaster neorectus & $47.3 / 51.2$ & & & & & $14-2 / 15.3$ & $13-5$ & \\
\hline & Discoaster neona matus & Discoaster bellus & $47-3 / 51-2$ & & & $1-5 / 2-2$ & $12-3$ & $14-2 / 15-3$ & & $24-2 / 29-5$ \\
\hline \multirow{5}{*}{$\begin{array}{l}\text { Middle } \\
\text { Miocene }\end{array}$} & Discoaster hamatus & & $53-2 / 55-2$ & & & & & $15-6$ & $14-5$ & $30-2 / 30-6$ \\
\hline & Catinaster coalitus & & ?56-2 & & & & & & & \\
\hline & \multirow{2}{*}{ Discoaster exilis } & Discoaster kugleri & $57-2 / 60-2$ & & & & $14-2$ & & \multirow{2}{*}{$15-2 / 16-5$} & \\
\hline & & Coccolithus miopelagicus & 61.4 & & & $4-2$ & & & & $32-1$ \\
\hline & Sphenolithus heteromorphus & & $62-1$ & & & & $15-4$ & $17-6$ & $17-5$ & $32-5 / 38-5$ \\
\hline \multirow{4}{*}{$\begin{array}{l}\text { Lower } \\
\text { Miocene }\end{array}$} & Helico pontosphaera a mpliaperta & & & & & & & & $18-5$ & $39-5 / 41-5$ \\
\hline & Sphenolithus belemnos & & & & & & & $? 18-3$ & $19-5$ & $42-4$ \\
\hline & & Discoaster druggii & & & & & & $19 \cdot 3$ & $20-5$ & \\
\hline & Triquetrorhabdulus carinatus & Discoaster deflandrei & & & & & & & & $43-2 / 48-2$ \\
\hline & & Cyclicargolithus abisectus & & & & $10-3$ & & $? 20-2$ & $21-2$ & \\
\hline & Sphenolithus ciperoensis & & & & & $12-2$ & & $21-5$ & $21-5 / 22-2$ & $49-2 / 51-4$ \\
\hline Oligogene & Sphenolithus distentus & & & & & & & $22-2 / 23-2$ & & $52-5 / 54-1$ \\
\hline Uigocene & Sphenolithus predistentus & & & & & & & $24-2 / 25-2$ & & \\
\hline & & Reticulofenestra hillae & & & & & & $26-2$ & & \\
\hline & Helicopontosphaera reticulata & Cyclococcolithina formosa & & & & & & $26-6 / 27-2$ & 23-2 & \\
\hline & & Coccolithus subdistichus & & & & & & & & \\
\hline Upper Eocene & Discoaster barbadiensis & & & & & & & $28-1$ & $23-3 / 23-6$ & \\
\hline & Reticulofenestra umbilice & Discoaster saipanensis & & & & & & & $24-1 / 26-4$ & \\
\hline & & Discoaster bifax & & & & & & & & \\
\hline & & Coccolithus staurion & & & & & & & $27-1 / 29-2$ & \\
\hline $\begin{array}{l}\text { Middle } \\
\text { Eocene }\end{array}$ & Nannotetrina quadrata & Chiasmolithus gigas & & & & & & & $29-5 / 31-1$ & \\
\hline & & Discoaster strictus & & & & & & & $32-1 / 33-3$ & \\
\hline & Discoaster sublodoensis & Rhabdosphaera inflata & & & & & & & & \\
\hline & & Discoasteroides kuepperi & & & & & & $29-1$ & $36-1$ & \\
\hline Lower & Discoaster lodoensis & & & & & & & $30-2 / ? 32-2$ & & \\
\hline Eocene & Tribrachiatus orthostylus & & & & & & & & & \\
\hline & Discoaster diastypus & & & & & & & & $37-2$ & \\
\hline & Discogster multiradiatus & Campylosphaera eodela & & & & & & & & \\
\hline & & Chiasmolithus bidens & & & & & & & $41-2$ & \\
\hline & Discoaster nohilis & & & & & & & & & \\
\hline & Discoaster mohleri & & & & & & & $33-3$ & $42-1 / 43-1$ & \\
\hline Paleocene & Heliolithus kleinpellii & & & & & & & & $43-3 / 44-2$ & \\
\hline & Fasciculithus tympaniformis & & & & & & & & $45 \cdot 1 / 51-2$ & \\
\hline & Cruciplacolithus tenuis & & & & & & & & $? 64-2 / ? 67-5$ & \\
\hline
\end{tabular}

Figure 2. Coccolith zonation of core samples from Leg 24. Numbers assigned to zones represent the cores and their 1.5-meter sections. Where samples from several sections are assigned to a single zone, the highest and lowest sections are listed with a slash between. Poorly diagnostic samples are assigned to an interval of several zones or are queried. At Site 238, Core 1 shows massive reworking and Cores 4 and 5 contain anomalously old assemblages suggesting, together, that the upper five cores are disturbed and stratigraphically unreliable. 